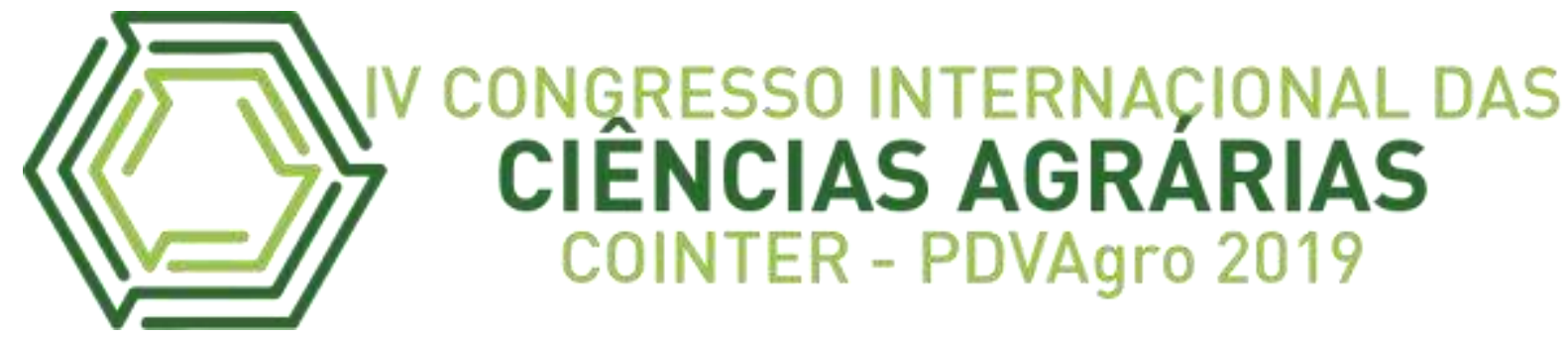

\title{
EFEITO DA DENSIDADE NO CRESCIMENTO DE Crassostrea gasar CULTIVADA EM SISTEMA FIXO NA COSTA DE MANGUEZAIS DE MACROMARÉS DA AMAZÔNIA, BRASIL
}

\section{EFECTO DE LA DENSIDAD EN CRECIMIENTO DE Crassostrea gasar CULTIVADA EN SISTEMA FIJO EN LA COSTA DE MANGLARES DE MACROMAREAS DE AMAZONÍA, BRASIL}

\section{EFFECT OF DENSITY ON GROWTH OF Crassostrea gasar FARMED IN A FIXED SYSTEM ON AMAZON MACROTIDAL MANGROVES COAST, BRAZIL}

\author{
Apresentação: Comunicação Oral \\ Ana Melissa de Moraes Câmara ${ }^{1}$; Paulo Protásio de Jesus²; Edivânia Oliveira Silva ${ }^{3}$ Josinete \\ Sampaio Monteles ${ }^{4}$; Izabel Cristina da Silva Almeida Funo ${ }^{5}$
}

DOI: https://doi.org/10.31692/2526-7701.IVCOINTERPDVAgro.2019.0008

\section{Resumo}

O presente trabalho avaliou o efeito da densidade de estocagem no crescimento de Crassostrea gasar em sistema fixo na Costa de Manguezais de Macromarés da Amazônia (CMMA). O cultivo foi iniciado com 4.320 ostras de 20 a $30 \mathrm{~mm}$ de altura da valva em travesseiro de malha $9 \mathrm{~mm}$. Os animais foram submetidos a três tratamentos $\left(\mathrm{T}_{1}-600\right.$ ostras $/ \mathrm{m}^{2}, \mathrm{~T}_{2}-800 \mathrm{ostras} / \mathrm{m}^{2}$ e $\mathrm{T}_{3}-1000$ ostras $/ \mathrm{m}^{2}$ ) com 4 repetições e cultivados durante 270 dias em um sistema de cultivo fixo (cama), no município de Bequimão, Maranhão. A cada coleta foi determinado o crescimento das ostras (da altura da concha e peso vivo), as variáveis ambientais e a ocorrência da fauna incrustante e vágil associada. Foram recrutadas pós-larvas em ambiente natural, através de coletores artificiais, que permaneceram submersos por um período de, aproximadamente, 60 dias. Os dados evidenciaram que os parâmetros produtivos foram significativamente influenciados em função das densidades de estocagem testadas (ANOVA, $\mathrm{p}<0,05)$. Nos tratamentos com maior densidade ( 800 ostras/ $\mathrm{m} 2$ e 1.000 ostras/ m2) registrouse crescimento (altura da concha e peso vivo) significativamente superior das ostras e menor incidência da fauna incrustante e vágil associada. Os meses com maior intensidade pluviométrica e com menor salinidade favoreceram para o menor crescimento das ostras, além de ter contribuído para a maior aglomeração de organismos indesejáveis ao cultivo. Assim, conclui-se que o crescimento da ostra nativa $C$. gasar apresentou diferença significativa em

\footnotetext{
${ }^{1}$ Licenciatura em Ciências Agrárias, IFMA - Campus São Luís Maracanã, m.moraes@acad.ifma.edu.br

${ }^{2}$ Licenciatura em Ciências Agrárias, IFMA - Campus São Luís Maracanã, paulo.protasio@acad.ifma.edu.br

${ }^{3}$ Técnico em Aquicultura, IFMA - Campus São Luís Maracanã, e-mail edivaniasilva@acad.ifma.edu.br

${ }^{4}$ Licenciatura em Biologia, IFMA - Campus Monte Castelo, josimonteles@gmail.com

${ }^{5}$ Doutora em Recursos Pesqueiros e Aquicultura, IFMA - Campus São Luís Maracanã, izabelfuno@ifma.edu.br
} 
função da densidade de estocagem testada, com altura da valva e peso vivo favorecidos em alta densidade. Sugere-se que a menor incidência de organismos competidores nas densidades mais altas pode ter contribuído para o melhor desempenho produtivo da ostra nesses tratamentos.

Palavras-Chave: Ostreicultura; Densidade; Parâmetros produtivos; Macromarés

\title{
Resumen
}

El presente trabajo evaluó el efecto de la densidad de población en el crecimiento de Crassostrea gasar en un sistema fijo en la Costa de Manglares de Macromareas de Amazonía (CMMA). El cultivo fue iniciado con 4.320 ostras de 20 a $30 \mathrm{~mm}$ de altura en la valva en almohada de malla de $9 \mathrm{~mm}$. Los animales fueron sometidos a tres tratamientos (T1 - 600 ostras $/ \mathrm{m}^{2}, \mathrm{~T} 2$ - 800 ostras $/ \mathrm{m}^{2}$ y T3 - 1000 ostras $/ \mathrm{m}^{2}$ ) con 4 repeticiones y se cultivaron durante 270 días en un sistema de cultivo fijo (basura) en la ciudad de Bequimão, Maranhão (Brasil). En cada cosecha, se determinó el crecimiento de la ostra (altura de la concha y el peso vivo), las variables ambientales y la ocurrencia de fauna incrustante y vágil asociada. Fueron cosechadas postlarvas en el medio natural a través de recolectores artificiales, que permanecieron sumergidos durante un período de aproximadamente 60 días. Los datos demostraron que los parámetros productivos fueron influenciados significativamente por las densidades de almacenamiento probadas (ANOVA, $\mathrm{p}<0.05$ ). En los tratamientos con mayor densidad de almacenamiento (800 ostras $/ \mathrm{m}^{2}$ y 1,000 ostras $/ \mathrm{m}^{2}$ ) hubo un crecimiento significativamente mayor (altura de la concha y peso vivo) de las ostras y una menor incidencia de fauna incrustante y vágil asociada. Los meses con mayor intensidad de lluvia y menor salinidad favorecieron el menor crecimiento de las ostras y contribuyeron para una mayor aglomeración de organismos indeseables en el cultivo. Por lo tanto, se puede concluir que el crecimiento de la ostra nativa C. gasar presentó una diferencia significativa en función de la densidad de almacenamiento probada, favoreciendo la alta densidad de la valva y el peso vivo. Se sugiere que la menor incidencia de organismos competidores en densidades más altas puede haber contribuido a un mejor rendimiento de la ostra en estos tratamientos.

Palabras Clave: Ostricultura; Densidad; Parámetros productivos; Macromareas.

\begin{abstract}
This work evaluated the effect of stocking density on the growth of Crassostrea gasar in a fixed system on Amazon Macrotidal Mangrove Coast (AMMC). The oyter farm was started with 4,320 oysters from 20 to $30 \mathrm{~mm}$ shell high on $9 \mathrm{~mm}$ mesh pillow. The animals were submitted to three treatments (T1 - 600 oysters $/ \mathrm{m} 2$, T2 - 800 oysters $/ \mathrm{m} 2$ and T3 - 1000 oysters $/ \mathrm{m} 2$ ) with 4 replications and farmed for 270 days in a fixed cultivation system (bed) in the Bequimão city, Maranhão. At each collect, the oyster growth (shell height and live weight), environmental variables and the occurrence of associated foul and fouling fauna were determined. Crassostrea gasar Larvae were recruited in the natural environment through artificial collectors, which remained submerged for a period of approximately 60 days. The analyzes showed that productive parameters were significantly influenced by the stocking densities tested (ANOVA, $\mathrm{p}<0.05$ ). In treatments with higher density ( 800 oysters $/ \mathrm{m} 2$ and 1,000 oysters $/ \mathrm{m} 2$ ) the oysters growth (shell height and live weight) was significantly higher and there was lower incidence of fouling and associated fauna. The months with higher rainfall and lower salinity favored the lower oysters growth and contributed to greater agglomeration of undesirable organisms. Thus, it can be concluded that growth of $C$. gasar, native oysters, showed significant difference as a function of the stocking density tested, with the shell height and live weight favored in high
\end{abstract}


density. It is suggested that lower incidence of competing organisms at higher densities may have contributed to better productive performance of oyster in these treatments.

Keywords: oyster farming; density; productive parameters; macrotidal.

\section{Introdução}

A produção aquícola mundial contribuiu com 110,2 milhões de toneladas no ano de 2016, sendo 15,5\% do volume global produzido, cerca de 17,1 milhões de toneladas, provenientes da malacocultura (cultivo de moluscos), a qual representa a quarta principal atividade da aquicultura mundial (FAO, 2018). A produção de moluscos bivalves se divide em quatro grupos principais: ostras, mexilhões, vieiras e "clams" (mariscos), que é como são conhecidos os moluscos de areia (FAO, 2015). Destes, a ostreicultura é considerada uma atividade sólida no campo da produção de alimentos de procedência aquática, somando no ano de 2017 uma produção de 5.710 .522 de toneladas (FAO, 2017).

De acordo com a FAO, o Brasil é o segundo maior produtor de moluscos bivalves da América Latina (FAO, 2017). Em 2017, a produção brasileira de moluscos bivalves cultivados (ostras, vieiras e mexilhões) foi de aproximadamente 20,9 mil toneladas, sendo que somente o estado de Santa Catarina deteve cerca de 98\% dessa produção (IBGE, 2017).

A ostreicultura já vem sendo praticada comercialmente há pouco mais de quatro décadas no Brasil e está vinculada a produção de três espécies, duas delas são endêmicas Crassostrea rhizophorae (Guilding 1828) e Crassostrea gasar (Adanson 1757 = Crassostrea brasiliana, Lamarck 1819), conhecidas popularmente como ostras do mangue, e uma exótica, a Crassostrea gigas (Thunberg 1793), conhecida como ostra japonesa. Embora a $C$. gasar e $C$. rhizophorae sejam amplamente utilizadas como alimento e como uma fonte de renda para a população local dos estuários brasileiros, tais espécies não estão bem representadas no cenário produtivo brasileiro, já que a $C$. gigas é a principal espécie cultivada (EPAGRI, 2014; FAO, 2014; IBGE, 2014).

Pesquisas destacam que as diferenças encontradas para o crescimento e sobrevivência das ostras cultivadas podem ser explicadas em função de vários fatores, tais como: condições climáticas; variáveis ambientais; espécie utilizada; genética das ostras; pela presença de organismos indesejáveis; sistema de cultivo e densidade de estocagem (HERNÁNDEZ et al., 1998; PEREIRA et al., 2001; LODEIROS et al., 2006; LOPES et al., 2013; PAIXÃO et al., 2013). 
A densidade de estocagem adequada na ostreicultura pode garantir melhores resultados no crescimento e sobrevivência das ostras cultivadas aumentando a produtividade, no entanto, esta densidade ideal pode variar de uma região para outra em função de suas condições ambientais (MACCACCHERO et al., 2007; CARDOSO-JÚNIOR et al., 2012; FUNO, 2016).

Ao longo do litoral brasileiro, há alguns estudos que avaliaram o crescimento e a sobrevivência das ostras nativas em condições de cultivo. Pereira et al. (2001) registraram taxa de crescimento mensal de $C$. gasar variando de 2,2 a 2,6 mm e sobrevivência de 64 a 90,1\% no final de dez meses de cultivo para a região estuarina de Cananéia, São Paulo. Funo (2016) cultivando a ostra C. gasar em áreas estuarinas no Estado do Maranhão, registrou taxa de crescimento mensal de $4,9 \mathrm{~mm}$ e $6 \mathrm{~mm}$ e sobrevivência de $45,7 \%$ e 51,3\% no final de sete meses de cultivo, para os sistemas de cultivo balsa e espinhel, respectivamente.

Ramos et al. (1986) avaliaram o crescimento da Crassostrea rhizophorae em Salina da Margarida, Bahia, onde foi observado um melhor crescimento desta espécie sob imersão contínua e um decréscimo na taxa de crescimento e da sobrevivência com o passar do tempo. Cardoso et al. (2006) avaliaram o crescimento da "ostra nativa" C. rhizophorae em diferentes densidades (50, 100 e 150 ostras/andar de lanterna) no município de Goiana, Pernambuco. E concluíram que a densidade de 400 ostras $/ \mathrm{m}^{2}$ (50 ostras/andar) apresentou o melhor resultado de produção, embora não tenham sido registradas diferenças significativas para o crescimento e sobrevivência das ostras durante o período de cultivo.

Deste modo, por conta da carência de trabalhos que avaliem a densidade de estocagem mais adequada para o cultivo de ostras nativas, especificamente para a espécie C. gasar são necessárias para o desenvolvimento da atividade de ostreicultura no litoral do Maranhão, considerando-se as características ambientais da região. Portanto, visando contribuir com o aprimoramento das técnicas de cultivo de ostras nativas ao longo do litoral maranhense, o objetivo do presente trabalho foi avaliar o efeito da densidade de estocagem no crescimento e sobrevivência da ostra nativa $C$. gasar cultivada na região estuarina do município de Bequimão, Maranhão.

\section{Fundamentação Teórica}

Em razão da crescente demanda de organismos aquáticos e da necessidade de suprimento do déficit deixado pela pesca extrativa, a aquicultura tem se tornado uma atividade cada vez mais importante (NONÔ, 2010). A maricultura (cultivo de organismos marinhos) 
desenvolvida apropriadamente e comunitariamente tem o potencial de recuperar a contribuição destes recursos marinhos e estuarinos para melhorar as condições de vida das populações menos assistidas do litoral, de maneira equitativa e sustentável - provendo particularmente oportunidades apropriadas para mulheres. A mudança da atitude extrativista tradicional para a de cultivo em fazendas marinhas, vem proporcionando renda adicional pela geração de emprego, além da fixação das populações tradicionais nas áreas de origem.

Os moluscos bivalves são organismos marinhos e o cultivo destes tem se comprovado como uma importante ferramenta para a diminuição da pobreza através da geração de emprego, renda e alimento em comunidades tradicionais costeiras frente à crescente diminuição dos recursos pesqueiros no Brasil. Tal atividade ajuda a fixar populações nativas litorâneas em seu ambiente tradicional e torna-se um complemento de renda para pescadores artesanais (OLIVEIRA, 2005). Também é uma atividade sustentável pois pode colaborar com a manutenção dos estoques naturais estuarinos, onde é realizada a maior parte da pesca artesanal (BALDAN; BENDHACK, 2009).

O cultivo de ostras nativas apresenta grande potencial para ser desenvolvido em vários pontos do litoral brasileiro, porém, necessita-se de mais pesquisas para aumentar a produtividade desta atividade (MIRANDA e GUZENSKI, 1999; TURECK, 2010). Nas regiões norte e nordeste vêm sendo cultivadas apenas as espécies $C$. rhizophorae e/ou $C$. gasar, e no ano de 2017 essas regiões juntas produziram cerca de 181 toneladas de ostras, representando uma pequena porcentagem $(0,9 \%)$ da produção nacional de moluscos bivalves, que inclui ostras, vieiras e mexilhões (IBGE, 2017).

A zona costeira maranhense possui $640 \mathrm{~km}$ de extensão e abriga um mosaico de ecossistemas de alta relevância ambiental. Ao longo do litoral alternam-se mangues, restingas, campos inundáveis, dunas, estuários, recifes de corais e outros ambientes importantes do ponto de vista ecológico. Diferentemente de outros estados brasileiros, a zona costeira maranhense revela uma expressiva ocorrência de manguezais relativamente preservados (UFMA, 2003). Esses ecossistemas devem ser explorados por projetos que visem o uso sustentável da biodiversidade marinha e estuarina pelo homem, gerando trabalho, renda e um melhor desempenho dos aspectos socioeconômicos da população ribeirinha.

Com o apoio de órgão financiadores de pesquisas, instituições de ensino (UFMA, IFMA e UEMA) e de algumas ações pontuais do governo, nos últimos anos tem se intensificado as pesquisas sobre as ostras nativas na costa maranhense, incluindo estudos sobre a biologia 
reprodutiva (SOUSA, 2015), genética (LOPES, 2018), cultivo (SANTOS, 2013; FUNO et al., 2013 e FUNO, 2016) e a seleção de áreas potenciais para a implantação do cultivo (FSADU, 2010; FRANÇA et al., 2013).

O crescimento e a sobrevivência das ostras nativas do gênero Crassostrea sofrem influência estatisticamente significativa da densidade de estocagem a que ela é submetida (BRUNETTO, 2018; FREIRE, 2018). Brunetto (2018) afirma que a altura da concha da ostra nativa $C$. gasar e sua sobrevivência são favorecidas em alta densidade, no entanto, o seu comprimento em baixa densidade. Freire (2018) avaliando o desempenho da ostra C. gasar sob as densidades de estocagem de 400, 600, 800 e 100 ostras $/ \mathrm{m}^{2}$, registrou crescimento médio mensal e peso médio estatisticamente superiores nas duas maiores densidades, e maior sobrevivência nas duas menores. Pereira et al. (2001) cultivando ostras da espécie $C$. brasiliana (=C. gasar), sob quatro densidades de estocagem registrou maior crescimento médio mensal e sobrevivência na maior delas.

Cardoso-Júnior et al. (2012), em experimento com a ostra nativa C. rhizophorae constatou um maior crescimento médio mensal das ostras cultivadas sob a menor densidade de estocagem, que foi de $400 \mathrm{ostras} / \mathrm{m}^{2}$. Modesto et al. (2010) avaliou o crescimento de ostras da espécie C. rhizophorae em travesseiros com três densidades de estocagem (250, 500 e 750 ostras/travesseiro), registrando a maior sobrevivência na menor densidade.

No Brasil, a realização de estudos sobre a exploração de moluscos comestíveis é muito importante, especialmente para o estado do Maranhão, que possui uma concentração considerável de espécies que servem para o consumo humano (Moreira, 2007; Monteles et al., 2009). Apesar de ter clima tropical e áreas favoráveis para o desenvolvimento da maricultura o estado do Maranhão ainda não apresenta índices representativos. Por meio de um determinado número de ações a nível institucional e governamental, seria possível fomentar o desenvolvimento da malacocultura sustentável neste estado (UFMA 2003).

Diante do exposto, são escassos os trabalhos publicados que tenham avaliado a influência da densidade de estocagem, sobre o crescimento e sobrevivência das ostras nativas ao longo do litoral brasileiro, especialmente para a espécie $C$. gasar em regiões sob regime de macromarés. Assim, pesquisas nessa área são necessárias para contribuir com o aprimoramento de metodologias de cultivo adequadas para a espécie, levando em consideração às características ambientais da região. 


\section{Metodologia}

Juvenis de ostras da espécie Crassostrea gasar foram obtidas através de coletores artificiais confeccionados de garrafa PET em um sementeiro localizado na comunidade de Lauro Sodré $\left(00^{\circ} 51^{\prime} 15,0^{\prime}\right.$ S e $\left.47^{\circ} 53^{\prime 2} 25,0^{\prime \prime} \mathrm{W}\right)$ no munícipio de Curuçá, Pará, Brasil. Posteriormente, as ostras foram transportadas para um estuário localizado no povoado de

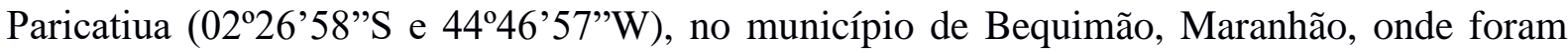
aclimatadas e posteriormente submetidas ao experimento.

O experimento foi realizado durante 270 dias (entre os meses de novembro de 2017 e setembro de 2018) era inteiramente casualizado com três tratamentos $\left(\mathrm{T}_{1}: 600\right.$ ostras $/ \mathrm{m}^{2} ; \mathrm{T}_{2}$ : 800 ostras $/ \mathrm{m}^{2} ; \mathrm{T}_{3}: 1.000$ ostras $/ \mathrm{m}^{2}$ ) e quatro repetições (travesseiros), perfazendo 12 unidades experimentais. Na primeira etapa deste experimento foram utilizadas ostras medindo entre 20 a $30 \mathrm{~mm}$, as quais foram acondicionadas em travesseiros ( $9 \mathrm{~mm}$ de malha) durante 3 meses. Na segunda etapa, quando as ostras alcançaram $40 \mathrm{~mm}$ de altura da concha, as mesmas foram remanejadas para travesseiros com abertura de malhas de $21 \mathrm{~mm}$ e foram mantidas na densidade de povoamento inicial, onde foram cultivadas por mais três meses.

A cada coleta, foi realizada a limpeza das malhas dos travesseiros e a biometria das ostras, na qual 30 organismos de cada unidade experimental (totalizando 360 por amostragem) foram medidos a altura das valvas $(\mathrm{mm})$, com auxílio de um paquímetro com precisão de 0,1 mm e pesados para determinação do peso vivo ( $\mathrm{g}$ ) com uma balança digital com precisão de três decimais. A padronização das medidas de biometria das valvas das ostras durante o experimento baseou-se no método proposto por Galtsoff (1964).

Paralelamente ao manejo era determinada a temperatura da água próximo da superfície utilizando um termômetro com precisão de $0,5^{\circ} \mathrm{C}$ e a salinidade através de um refratômetro. Os dados de precipitação pluviométrica para o município de Raposa foram cedidos pelo Núcleo de Geoprocessamento da Universidade Estadual do Maranhão.

Durante as amostragens, também foi realizada a coleta dos organismos incrustantes e associados (fauna vágil) presentes na tela das estruturas de cultivo e no interior dos travesseiros. O material coletado foi fixado com formalina a $4 \%$ por 24 horas, e em seguida foi transportado até ao laboratório NUMAR do IFMA - Campus Maracanã, a fim de ser congelado para preservar e manter as características naturais dos organismos, possibilitando o processo de identificação dos táxons. 
As análises estatísticas foram realizadas no programa Statistica 7.0 (StatSoft Inc., USA). O teste paramétrico one-way ANOVA foi utilizada para avaliar o efeito da densidade sobre os parâmetros de produção (altura da concha e peso vivo) após a confirmação da homocedasticidade (Cochran $\mathrm{P}<0,05)$ e normalidade (Shapiro-Wilk $\mathrm{P}<0,05)$. O teste de Tukey $(\mathrm{P}<0,05)$ foi realizado para comparação e classificação significativa a partir dos três tratamentos.

\section{Resultados e Discussão}

Ao final dos 270 dias de experimento as ostras alcançaram a altura média da concha de $44,9 \pm 4,7 \mathrm{~mm}, 53,8 \pm 4,3 \mathrm{~mm}$ e $54,4 \pm 3,9 \mathrm{~mm}$. A taxa de crescimento médio mensal foi de 2,69 $\mathrm{mm}, 3,12 \mathrm{~mm}$ e $3,19 \mathrm{~mm}$ nos tratamentos $\mathrm{T}_{1}\left(600\right.$ ostras $\left./ \mathrm{m}^{2}\right), \mathrm{T}_{2}\left(800\right.$ ostras $\left./ \mathrm{m}^{2}\right)$ e $\mathrm{T}_{3}(1000$ ostras $/ \mathrm{m}^{2}$ ), respectivamente. Até os 45 dias de experimento, as diferenças entre as medidas de altura dos tratamentos não foram significativas $(\mathrm{P}<0,05$, Tukey). Porém, a partir da terceira biometria (aos 90 dias de cultivo), os valores encontrados de altura no tratamento $\mathrm{T}_{1}$ mostraramse significativamente menores $\left(\mathrm{P}<0,05\right.$, Tukey) que os valores encontrados nos tratamentos $\mathrm{T}_{2}$ e $\mathrm{T}_{3}$, que por sua vez, não apresentaram diferenças significativas entre si (Figura 1).

Figura 1. Valores médios de altura de concha da ostra Crassostrea gasar exposta à diferentes densidades de estocagens no estuário de Paricatiua - MA. T1 - Tratamento 1, T2 - Tratamento 2 e T3 - Tratamento 3.

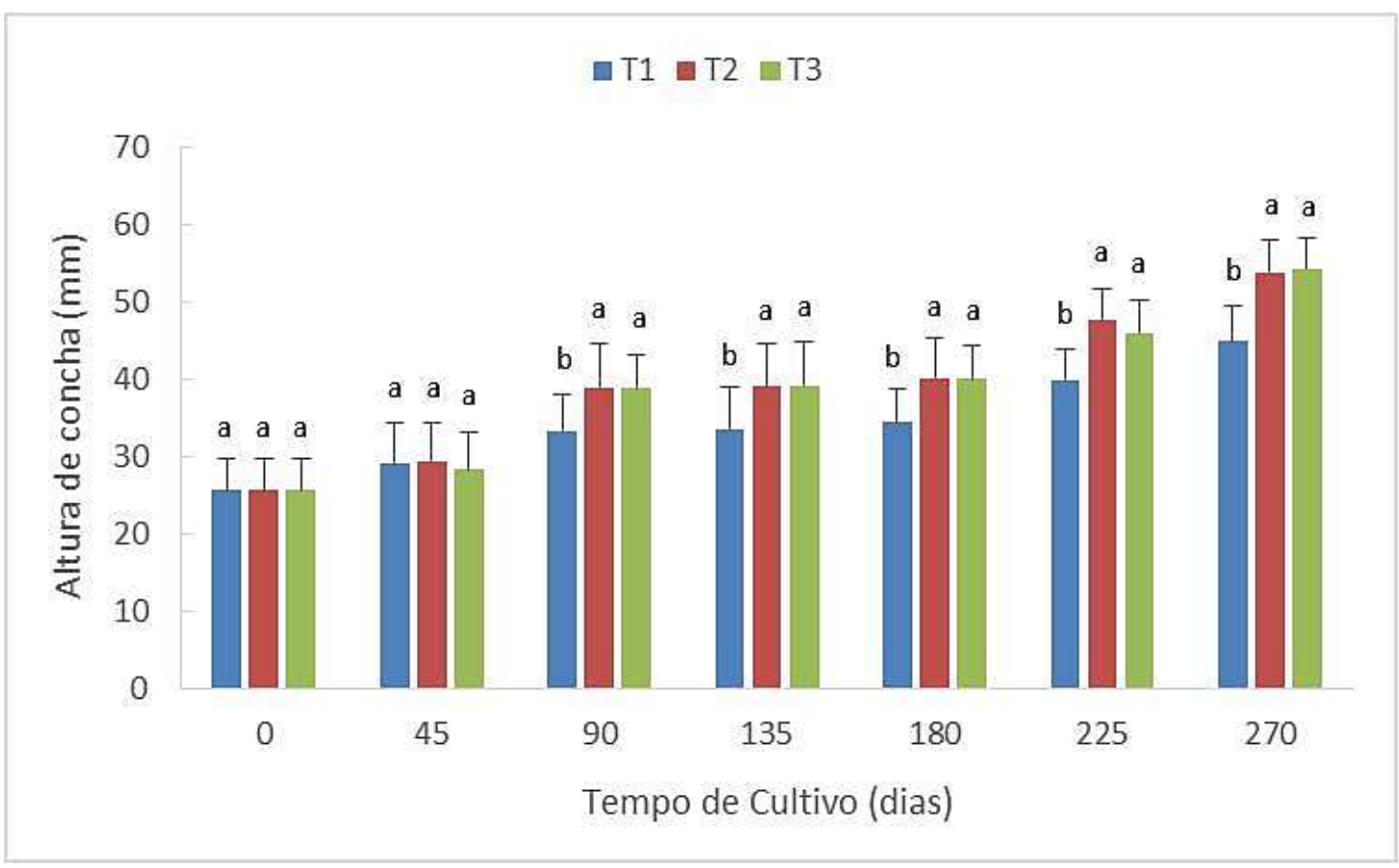


No presente trabalho, as ostras cultivadas na maior densidade apresentaram um maior crescimento médio mensal, corroborando com os resultados obtidos no estudo realizado por Freire (2018), que em pesquisa realizada no município de Primeira Cruz - MA, cultivando a ostra nativa Crassostrea gasar em travesseiros em sistema de cultivo suspenso fixo e submetendo-as às densidades de estocagem de 400, 600, 800 e 1000 ostras $/ \mathrm{m}^{2}$, obteve taxas de crescimento médio mensal variando de $1,06 \pm 1,91 \mathrm{~mm}$ a 2,42 $22,58 \mathrm{~mm}$, com valores significativamente superiores nas duas maiores densidades testadas.

Estudo realizado por Brunetto (2018), em sistema de cultivo suspenso flutuante (longline) evidenciou que a alta densidade de estocagem em cultivo influencia positivamente na altura de concha das ostras da espécie Crassostrea gasar, corroborando com os dados do presente trabalho. Vale ressaltar que as taxas de crescimento médio mensal registradas neste estudo para as densidades de 600, 800 e 1000 ostras $/ \mathrm{m}^{2}$ foram superiores às registradas no trabalho de Freire (2018), tal fato pode ser justificado por serem estuários com condições ambientais diferentes.

No entanto, Cardoso-Júnior et al. (2012) observou em experimento realizado com a espécie C. rhizophorae em Goiana - PE, uma taxa de crescimento maior para as ostras cultivadas na menor densidade, de 400 ostras $/ \mathrm{m}^{2}$, onde estas apresentaram um crescimento médio mensal de $5,53 \mathrm{~mm}$. No estudo realizado por estes autores as ostras apresentaram uma taxa de crescimento médio mensal superior à do presente estudo, tal fato pode ser atribuído ao sistema de cultivo adotado na pesquisa, eles utilizaram lanternas alocadas em sistema suspenso flutuante balsa, no qual as ostras ficaram sempre submersas, possuindo assim disponibilidade de alimento a todo o tempo.

Em experimento realizado na cidade de Cananéia - SP, em sistema suspenso fixo foram testadas quatro densidades em três locais diferentes, as ostras cultivadas na maior densidade, 25 dúzias ostras $/ \mathrm{m}^{2}$, apresentaram o melhor crescimento médio mensal nesses três locais $(2,64$ $\mathrm{mm}, 2,16 \mathrm{~mm}$ e $2,18 \mathrm{~mm}$ ), embora a diferença entre o crescimento nas outras densidades testadas não tenha sido estatisticamente significante (PEREIRA et al., 2001). As taxas de crescimento média mensal registrada no presente estudo variaram de 2,69 $\mathrm{mm}$ a 3,19 $\mathrm{mm}$, ou seja, valores superiores aos registrados no experimento realizado por Pereira et al. (2001).

Os resultados do presente estudo evidenciaram que o peso dos organismos cultivados foi influenciado significativamente em função da densidade de estocagem, sendo que as ostras cultivadas nas maiores densidades $\left(\mathrm{T}_{2}\right.$ e $\left.\mathrm{T}_{3}\right)$ apresentaram ganho de peso estatisticamente 
superior $(\mathrm{P}<0,05$, Tukey). Vale ressaltar que essa diferença estatística ocorreu a partir 135 dias de cultivo (quarta biometria), e tal comportamento se manteve até o término do experimento (Figura 2).

Figura 2. Valores médios de ganho de peso da ostra Crassostrea gasar exposta à diferentes densidades de estocagens no estuário de Paricatiua - MA. T 1 - Tratamento 1, $\mathrm{T}_{2}-$ Tratamento 2 e $\mathrm{T}_{3}-$ Tratamento 3.

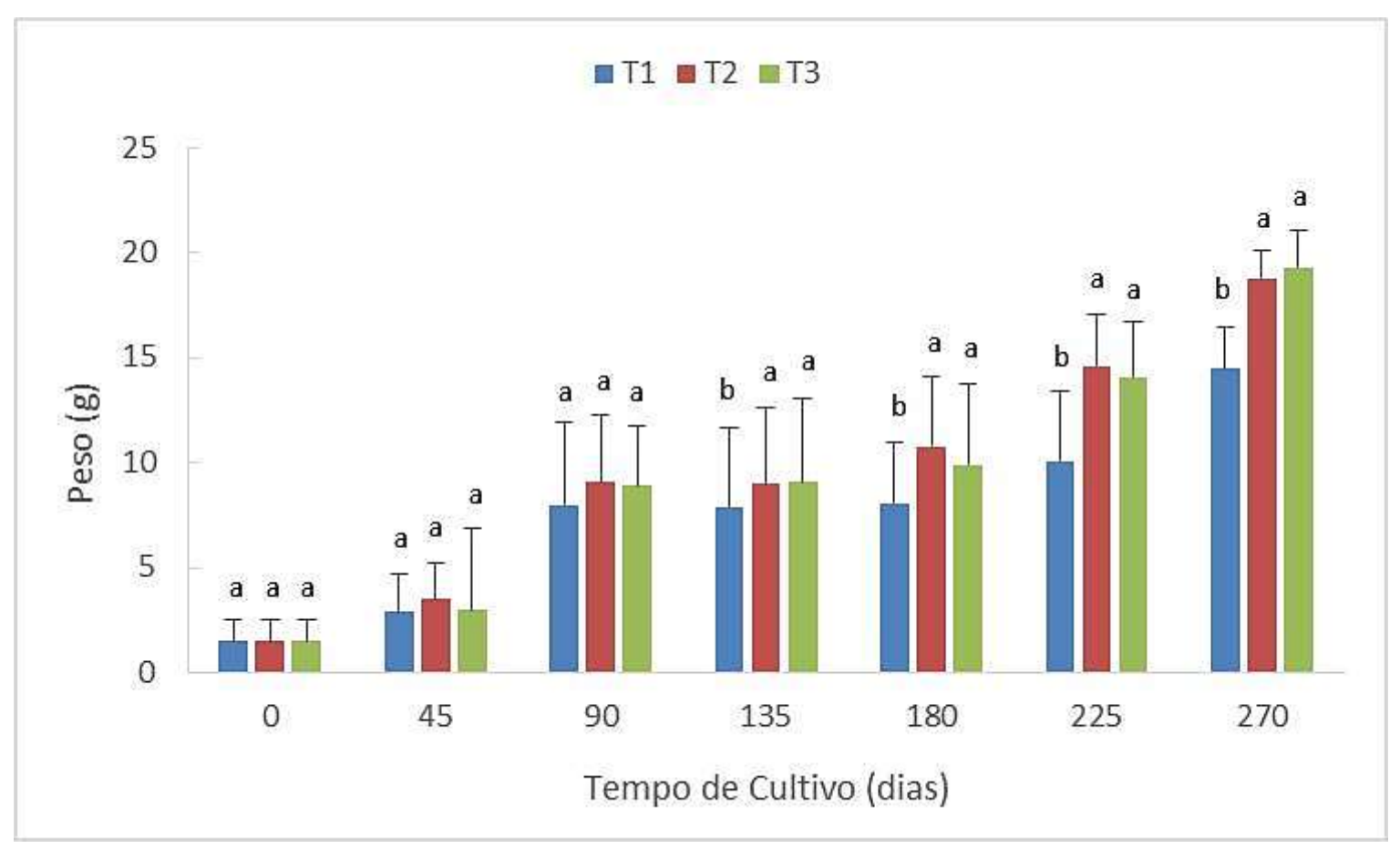

Freire (2018) cultivando a mesma espécie no município de Primeira Cruz (Maranhão), obteve resultados semelhantes, pois cultivando da mesma forma a ostra nativa $C$. gasar em sistema suspenso fixo e submetendo-as às densidades 400, 600, 800 e 1000 ostras $/ \mathrm{m}^{2}$ registrou no final do cultivo, maiores valores de peso médio nas maiores densidades de estocagem $\left(22,73 \pm 7,28 \mathrm{~g} \mathrm{e} 19,01 \pm 7,62 \mathrm{~g}\right.$ para as densidades de 800 e $1000 \mathrm{ostras} / \mathrm{m}^{2}$, respectivamente). Pesquisa realizada Brunetto (2018), testou diferentes densidades de estocagem em sistema de cultivo suspenso flutuante (long-line) para a ostra Crassostrea gasar e os resultados indicaram maior ganho de peso nas ostras cultivadas sob maior densidade na fase definitiva (180 ostras/andar de lanterna).

No presente estudo, durante o período experimental, a salinidade da água variou de $5,0 \pm 3,5$ a $31,0 \pm 3,5 \mathrm{~g} \cdot \mathrm{Kg}^{-1}$; a temperatura de $28,0 \pm 0,6$ a $29,3 \pm 1,1^{\circ} \mathrm{C}$ e a precipitação de 0 a 805,5 mm. Avaliando os resultados das variáveis ambientais em função do crescimento das ostras durante o período experimental, constatou-se que nos meses de maior precipitação 
pluviométrica e menor salinidade ocorreu crescimento mensal (altura da concha e peso vivo das ostras) significativamente inferior, quando comparado com os valores obtidos nos outros períodos de amostragem. Estudo realizado por Funo et al. (2015) constatou que o crescimento das ostras C. gasar é afetado pela salinidade, apresentando melhor desempenho em salinidades variando de 15 a 35. Brito (2008) cultivando ostras Crassostrea rhizophorae em laboratório e submetidas a salinidades de 20,25, 30 e 35 obteve melhor resultado de crescimento ao final de 150 dias de cultivo, na salinidade 25 .

Nos primeiros 30 dias de cultivo a salinidade oscilou de 0 a $31 \mathrm{~g} \cdot \mathrm{Kg}^{-1}$, coincidindo com maior fixação de cracas (Amphibalanus sp.) e ocorrência do parasita poliqueta (Alitta succinea) nas ostras cultivadas de todos os tratamentos testados, no entanto, quanto maior era a densidade menor era incidência desses organismos. Posteriormente, no período de 90 a 180 dias de cultivo, a pluviosidade da região foi elevada $(198,1$ a $805,5 \mathrm{~mm})$, nesta ocasião foi registrada diminuição da salinidade (10 a 26 g. $\mathrm{Kg}^{-1}$,) e uma intensa infestação de sementes de sururu (competidor) da espécie Mytella charruana fixadas nas estruturas de cultivo e nas conchas das ostras cultivadas. Outros organismos foram observados esporadicamente associados às estruturas de cultivo, como a ascídias, planárias e pequenos caranguejos.

Pesquisas evidenciaram que o "fouling", que cresce nas paredes e malhas das estruturas de cultivo, pode influenciar no rendimento do produto final, como o trabalho realizado por Hernández et al. (1998), o qual evidenciou que os organismos incrustantes (macroalgas, bivalves, ascídias, cracas, briozoários, hidrozoários e esponjas) fixados nas malhas das estruturas de cultivo reduzem a circulação, oxigenação e o aporte de partículas suspensas na água no interior dessas estruturas de cultivo, afetando o crescimento das ostras cultivadas.

Em todas as densidades avaliadas, a taxa de crescimento mensal (altura da concha e peso vivo) foi significativamente menor aos 135 dias de cultivo $(\mathrm{p}<0,5)$, coincidindo com o período que houve elevada aglomeração de sementes de $M$. charruana fixadas nas estruturas de cultivo e nas valvas das ostras. Funo (2016) constatou que os mitilídeos (M. charruana e $M$. guyanensis) sintetizam sua estrutura de fixação (bisso), aderindo-se nas redes e pratos das lanternas, e que esses filamentos de bisso, por serem pegajosos, formavam aglomerações de lodo no interior das lanternas, tal comportamento interferia no processo de alimentação das ostras e consequentemente no seu crescimento.

Monteles et al. (2018) avaliaram o recrutamento de sementes de sururu (Mytella charruana) na região estuarina de Paricatiua (Bequimão - MA), e concluíram que o 
recrutamento de semente desta espécie é sazonal, ocorrendo a fixação da semente nos coletores no período de maior precipitação pluviométrica e menor salinidade. Assim, no presente estudo constatou-se que a grande fixação de semente de $M$. charruana no cultivo de ostra pode afetar os parâmetros produtivos das ostras cultivadas.

Os resultados obtidos no presente estudo evidenciaram que a densidade de estocagem influencia no crescimento da ostra $C$. gasar, com crescimento significativamente superior nas maiores densidades, onde foram registrado menor incidência de organismos incrustantes. Estudos comprovaram que a adoção de altas densidades no cultivo desta espécie tem proporcionado maior crescimento da ostra, uma vez que as estruturas de cultivo com maior quantidade de ostras proporcionam menor competição por espaço e alimento com os organismos incrustantes (BRUNETTO, 2018; FREIRE, 2018; PEREIRA et al., 2001).

\section{Conclusões}

O crescimento de $C$. gasar apresentou diferença significativa em função da densidade de estocagem. A altura da concha e o peso vivo foram favorecidos em alta densidade (800 ostras/ $\mathrm{m}^{2}$ e 1.000 ostras/ $\mathrm{m}^{2}$ ), devido à menor interferência da fauna incrustante e vágil associada nas estruturas de cultivo.

Os meses com maior intensidade pluviométrica e com menor salinidade favoreceram para o menor crescimento das ostras, além de ter contribuído para a maior aglomeração da fauna incrustante e vágil associada.

\section{Referências}

BALDAN, A. P.; BENDHACK, F. MARICULTURA SUSTENTÁVEL NO LITORAL DO PARANÁ, BRASIL: atualidades e perspectivas. Rev. Acad., Ciênc. Agrár. Ambient., Curitiba, v. 7, n. 4, p.491-497, out. 2009.

BRITO, L. de. Efeito da salinidade sobre o crescimento da ostra nativa Crassostrea sp. como subsídio ao desenvolvimento da maricultura de espécies nativas em mar aberto. 2008. $38 \mathrm{f}$. Dissertação (Mestrado) - Curso de Sistemas Costeiro e Oceânico, Setor de Ciências da Terra, Centro de Estudos do Mar, Universidade Federal do Paraná, Pontal do Paraná, 2008.

BRUNETTO, L. J. CULTIVO DA OSTRA Crassostrea gasar EM DIFERENTES DENSIDADES. 2018. 71 f. Dissertação (Mestrado) - Curso de Aquicultura, Centro de Ciências Agrárias, Universidade Federal de Santa Catarina, Florianópolis, 2018.

CARDOSO, L. O.; Galvez, A. O. Avaliação do crescimento da C. rhizophorae (Guilding, 1828). 2006. 40p. Monografia (Graduação) - Universidade Federal Rural de Pernambuco, Recife. 
CARDOSO-JÚNIOR, L.O.; LAVANDER, H.D.; NETO, S.R.S.; SOUZA, A.B.S.; SILVA, L.O.B.; GÁLVEZ, A.O. Crescimento da ostra Crassostrea rhizophorae cultivada em diferentes densidades de estocagem no Litoral Norte de Pernambuco. Revista de Pesquisa Agropecuária, v.17, n.1, p.10-14, 2012.

EPAGRI. Empresa de Pesquisa Agropecuária e Extensão Rural de Santa Catarina. Síntese Informativa da Maricultura. Florianópolis, 2014. Disponível em: http://www.epagri.sc.gov.br/wpcontent/uploads/2013/08/Sintese_informativa_da_maricultura _2014.pdf. Acesso em: 5 set 2018.

FAO - Fisheries and Aquaculture Information and Statistics Branch, 2017. Disponível em: http://www.fao.org/fishery/statistics/global-aquaculture-production/query/en. Acesso em 24 de junho de 2019.

FAO - Food and Agriculture Organization of the United Nations. The State of World Fisheries and Aquaculture 2018: Meeting the sustainable development goals. Rome: FAO, 2018.

FAO. Fisheries Department, Fishery Information, Data and Statistics Unit. Fisheries and aquaculture software. FishStatJ: software for fishery statistical time series. Version 2.3. Rome, 2015. Disponível em: http://www.fao.org/fishery/statistics/software/en. Acesso em: $9 \mathrm{dez}$ 2018.

FAO. Fisheries Department, Fishery Information, Data and Statistics Unit. FishStat Plus: Universal Software For Fishery Statistical Time Series. Version 2.3. Rome, 2014. Disponível em: http://www.fao.org/fishery/statistics/software/en. Acesso em: 2 setembro 2018.

FRANÇA, V.L. de; MONTELES, J.S.; ALMEIDA FUNO, I.C.S.; CASTRO, A.C.L. de. Seleção de áreas potenciais para o cultivo de Ostra nativa, Crassostrea spp. e Sururu, Mytella falcata, em Raposa, Maranhão. Arquivo Ciências do Mar, v.46, n.1, p.62-75, 2013.

FREIRE, T. B. Efeito da densidade de estocagem no crescimento e sobrevivência da ostra nativa Crassostrea gasar (Adanson, 1757) cultivada em ambiente de macromaré de Primeira Cruz - MA. 2018. 43 folhas. Monografia (Graduação) - Curso de Engenharia de Pesca, Universidade Estadual do Maranhão, São Luís, 2018.

FSADU. Fundação Sousândrade de Apoio. Plano Local de Desenvolvimento da Maricultura - PLDM's do Maranhão: Icatu, Humberto de Campos e Primeira Cruz. São Luís, 2010. Disponível em: http://berbeladomar.bio.br/documentos/ RELATORIO_PLDM_2010/RELATORIO_PLDM_2010.pdf. Acesso em: 26 junho 2019.

FUNO, I. C. da S. A. Avaliação de parâmetros produtivos e biológicos da ostra nativa Crassostrea gasar (ADANSON, 1757) como subsídio ao desenvolvimento da ostreicultura em ambientes estuarinos do Estado do Maranhão. 2016. 122 f. Tese (Doutorado) -Recursos Pesqueiros e Aquicultura - Universidade Federal Rural de Pernambuco. Recife, 2016. 
FUNO, I. C. da S. A. et al. Influência da salinidade sobre a sobrevivência e crescimento de Crassostrea gasar. Bol. Inst. Pesca, São Paulo, v. 41, n. 4, p.837-847, 2015.

FUNO, I. C. da S. A.; ANTONIO, I.G.; MONTELES, J.S.; PEREIRA, A.J.; GUTERRES, I.R.; GALVEZ, A.O. Crescimento e mortalidade da ostra nativa Crassostrea gasar no município de Raposa, Maranhão. In: Congresso Brasileiro de Aquicultura de Espécies Nativa, 4., 2013, Belém. Anais. Belém/PA. UFPA, 2013. p. 12-12.

HERNÁNDEZ, O. D.; TROCCOLI, G.; J. MILLÁN, Q. Crecimiento, Engorde y Sobrevivencia de la Ostra de Mangle Crassostrea rhizophorae Guilding, 1828 en la Isla de Cubagüa, Venezuela. Caribbean Journal of Science, Mayagüez, v. 34, n. 1, p. 243-249, 1998.

IBGE - INSTITUTO BRASILEIRO DE GEOGRAFIA E ESTATÍSTICA. 2014. Pesquisa da Pecuária Municipal. Disponível em: https://sidra.ibge.gov.br/tabela/3940. Acesso em: 22 jun 2019.

IBGE - INSTITUTO BRASILEIRO DE GEOGRAFIA E ESTATÍSTICA. 2017. Pesquisa da Pecuária Municipal. Disponível em: https://sidra.ibge.gov.br/tabela/3940. Acesso em: 22 jun 2019.

LODEIROS, C.; BUITRAGO, E.; GUERRA, A. Evaluación del tipo de cestos de cultivo para la ostra de mangle Crassostrea rhizophorae suspendidas em long line y balsa. Ciencias Marinas, v.32, n.2, p.331-337, 2006.

LOPES, G. R.; GOMES, C.H.A.M.; TURECK, C.R.; MELO, C.M.R. Growth of Crassostrea gasar cultured in marine and estuary environments in Brazilian waters. Pesquisa Agropecuária Brasileira, v.8, n.7, p.975-982, 2013.

LOPES, R. G. P. S. et al. Molecular identification of native oysters on the coast of Maranhão, Brazil. Boletim do Instituto de Pesca, [s.1.], v. 44, n. 4, p.377, 21 set. 2018.

MACCACCHERO, G. B.; FERREIRA, J.F.; GUZENSKI, J. Influence of stocking density and culture management on growth and mortality of the mangrove native oyster Crassostrea sp. in southern Brazil. Biotemas, v.20, p.47-53, 2007.

MIRANDA, M. B. B. de; GUZENSKI, J. Cultivo larval da ostra do mangue, Crassostrea rhizophorae (Guilding, 1828), em diferentes condições de temperatura, salinidade e densidade. Arq. Ciên. Mar., Fortaleza, n. 32, p.73-84, 1999.

MODESTO, G. A. et al. Utilização de Crassostrea rhizophorae (Guilding 1828) no tratamento dos efluentes do cultivo de Litopenaeus vannamei (Boone 1931). Pan-american Journal Of Aquatic Sciences, [s.i.], v. 5, n. 3, p.367-375, jan. 2010.

MONTELES, J. S. et al. Percepção sócio-ambiental das marisqueiras no município de Raposa, Maranhão, Brasil. Rev. Bras. Eng. Pesca, v.4, n. 2, p. 34-45, 2009. 
MONTELES, J. S. et al. Recrutamento de semente do sururu Mytella falcata em manguezais de macromaré no litoral maranhense, usando materiais reciclados. In: VII AQUACIÊNCIA. Anais. Natal. 2018.

MOREIRA, I.C.N. 2007. Impactos do extrativismo da Anomalocardia brasiliana (Gmelin, 1791) nos estuários dos Rios Paciência e Cururuca, São Luís, Maranhão: uma visão etnoconservacionista. Dissertação (Mestrado). Universidade Federal do Maranhão, São Luís, Brasil.

NONÔ, Ricardo Gomes de Barros. Cultivo de Ostras em Alagoas. Maceió: Sebrae/al, 2010.

OLIVEIRA, B. de L. Impacto da Mitilicultura no desenvolvimento das comunidades tradicionais ao entorno das Praias da Cerca e Guaibura, Guarapari, ES. 2005. 67 f. Monografia (Especialização) - Curso de Oceanografia, Universidade Federal do Espírito Santo, Vitória, 2005.

PAIXÃO, L.; FERREIRA, M. A.; NUNES, Z.; FONSECA-SIZO, F.; ROCHA, R. Effects of salinity and rainfall on the reproductive biology of the mangrove oyster (Crassostrea gasar): Implications for the collection of broodstock oysters. Aquaculture, v.6, n.12, p.380-383, 2013.

PEREIRA, O. M.; MACHADO, I. C.; HENRIQUES, M. B.; YAMANAKA, N. Crescimento da ostra Crassostrea brasiliana semeada sobre tabuleiro em diferentes densidades na região estuarino-lagunar de Cananéia-SP $\left(25^{\circ} \mathrm{S}, 48^{\circ} \mathrm{W}\right)$. Boletim do Instituto de Pesca, v.27, p.163$174,2001$.

RAMOS, M. I. S.; NASCIMENTO, I.A; SILVA, J. DE L. The comparative growth survival of pacific oyster and C. rhizophorae in Todos os Santos Bay, Brazil. Ciência e Cultura, v.38, p.1604-1615, 1986.

SANTOS, G.M. Caracterização das marisqueiras e cultivo experimental de Crassostrea gasar no município de Raposa-MA. 2013. 51p. Monografia (graduação) - Universidade Estadual do Maranhão, São Luís.

SOUSA, A.K.R. Biologia reprodutiva da "ostra nativa" Crassostrea rhizophorae na ilha do maranhão - MA. 2015. 30p. Monografia (Graduação) - Universidade Estadual do Maranhão, São Luís.

TURECK, T.R. Sementes de ostras nativas no litoral de Santa Catarina/Brasil, como subsidio ao cultivo. 2010. 140p. Dissertação (Mestrado). Universidade Federal de Santa Catarina, Florianópolis.

UFMA/DEPARTAMENTO DE OCEANOGRAFIA E LIMNOLOGIA. Zoneamento Costeiro do Estado do Maranhão. 2003, 242p. 